\title{
Equity and Inclusion in Sanitation and Hygiene in South Asia: A Regional Synthesis
}

\author{
Ravi Narayanan, Hendrik van Norden, Louisa Gosling and \\ Archana Patkar
}

\begin{abstract}
Today, 2.6 billion people in the world have nowhere safely to defecate on a daily basis or to follow hygienic practices that are important for their health and wellbeing. People are different and require support to overcome the specific impediments that stand in the way of their being able to use services sustainably. There is a greater likelihood of success if we focus on the forgotten millions, first. When pastoralists, ethnic or religious minorities, the disabled, the chronically ill, children, the aged, adolescent girls, women, or anyone without voice or agency are centre stage, their needs are reflected in design and investment decisions, with gains for all, including the larger community. To make this happen for all those without sustainable sanitation and hygiene, we will need to redefine policy and practise so that equity is woven into the fabric of every investment, every supervisory mission, every reward and every audit.
\end{abstract}

\section{Introduction}

At the start of the second decade of the twentyfirst century, we are still faced with the shameful fact that in South Asia a staggering 716 million men, women and children defecate in the open every day, contributing to the most appalling concentration of poverty and disease, and the poorest standards of hygiene in the world; this in a region of galloping gross domestic product (GDP) and high growth rates. Who are these people and why are they left out, not reached or not served, why do they not use facilities or practise the key behaviours so critical for their own health, that of their neighbours and wellbeing in the region?

The collaborative process of desk review, consultations and analysis behind this article reinforces what we already know - that the 'excluded' are not only people who suffer from 'asset poverty', but also those who are shut out for social reasons. We also know that mere concentration on the big numbers will only serve to increase the gap between the haves and the have-nots, as gains are quickly snapped up by the better informed, better connected and better off, marginalising even further those who are left behind without services.

Business as usual, without explicit attention to equity, will result in more of the same, i.e. large numbers of people accessing services while large numbers - who also happen to be the most disadvantaged - continue to be left out. Put simply, success in South Asia would mean that everyone is able to practise safe sanitation and hygiene. This will require a paradigm shift in the way we measure success in South Asia. It will mean that equity and inclusion is the lens through which we view all progress in sanitation and hygiene, rather than a corollary that is often forgotten in the race to achieve results.

\section{The problem}

There are two facets to the problem in South Asia, both of which are unacceptable. The first is a problem of scale. Hundreds of millions of people in South Asia have historically practised open defecation, especially in rural areas: a veritable sanitation crisis that impairs progress in the region. Many districts in India, Nepal and Pakistan fall into this category. 


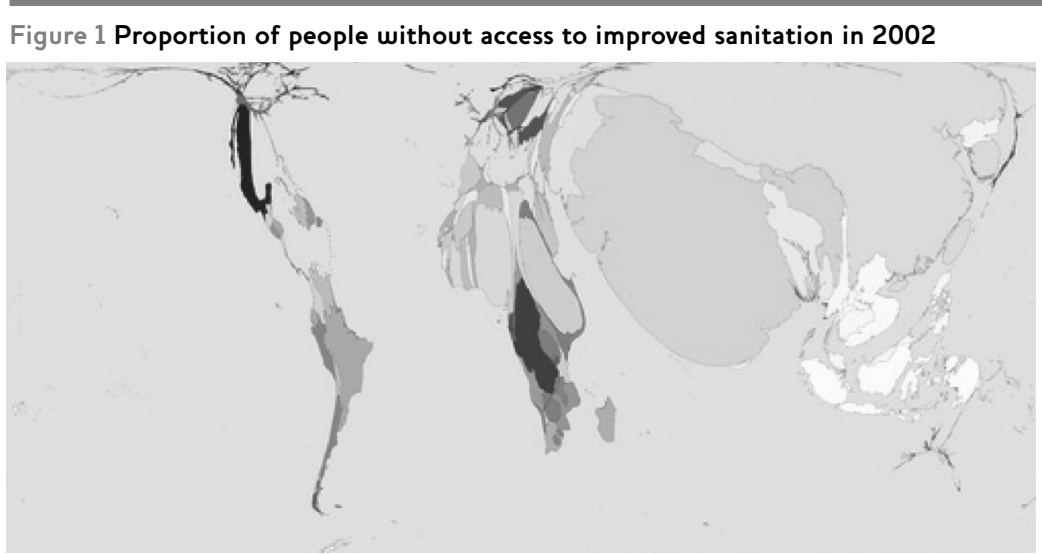

Note Territory size shows the proportion of all people without access to basic sanitation (toilets) that live there. The greys used on the maps group the territories into 12 geographical regions, and allow for an easier visual comparison between the maps than would otherwise be possible. The shading of each territory within a region is consistent throughout all of the maps.

Source www.worldmapper.org/index_map.html, www.worldmapper.org/region_map.html

Figure 2 Use of sanitation facilities 2008; circle size represents the equivalent population size

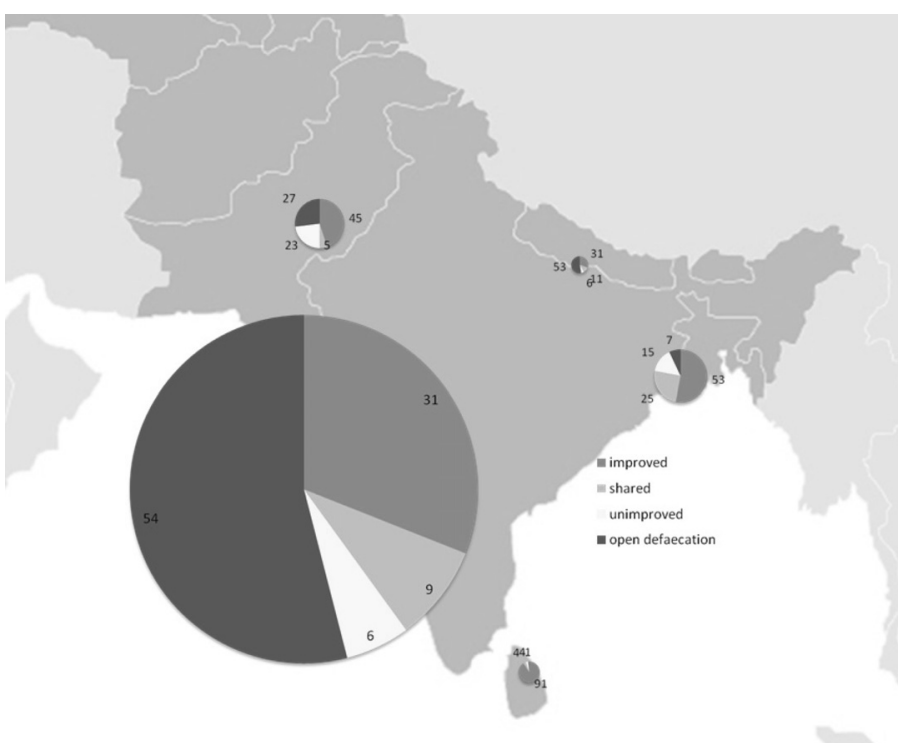

Source www.wssinfo.org/fileadmin/user_upload/resources/SACOSAN-Final.pdf (accessed 13 January 2012). Prepared using data from the JMP 2010 report, www.wssinfo.org/fileadmin/user_upload/resources/1278061137-JMP_report_ 2010_en.pdf (accessed 13 January 2012).

The second and in many ways more pernicious problem, particularly in South Asia, is one of exclusion, where different categories of people are not able to access and use safe sanitation facilities because they are socially and economically marginalised or excluded, or cannot use standard designs.

Data and analysis from the region show that the gains in sanitation have been primarily concentrated in the richer segments of the population. Figure 3 shows that the poorest quintile is 20 times less likely to have access to improved sanitation than the richest quintile.

The 'excluded' are not only people who suffer from 'asset poverty', but also those who are shut out for social reasons. The combination of economic and social exclusion creates subhuman 
living conditions in urban slums and rural areas across South Asia. Mere concentration on reaching the big numbers will only serve to increase the gap between the haves and the have-nots. It is this second problem that is often overlooked in South Asia and needs special and urgent attention.

\section{The imperative}

All governments in South Asia have voted in favour of a 2010 UN General Assembly resolution, declaring sanitation to be a human right. ${ }^{1}$ These countries have also committed themselves to the Millennium Development Goals (MDGs) but have failed to meet their targets. Children, especially those in poor families, pay the price: 570,000 young children die in South Asia every year as a result of diarrhoea caused by poor sanitation and hygiene. There is an indisputable moral imperative for action backed up by strong evidence of the huge economic costs associated with neglecting sanitation and the resulting burden of disease. In India, where 638 million people defecate in the open, a recent study by the Water and Sanitation Program (WSP 2011) in New Delhi estimated that inadequate sanitation costs the country the equivalent of 6.4 per cent of GDP (US\$53.8 billion), in terms of avoidable household and public health expenditure, losses in productivity from morbidity, and opportunity costs from loss of time. Despite all the apparent economic growth, the full potential of the energy and creativity of the peoples of South Asia will not be realised if open defecation is not eliminated.

Conventional wisdom states that more lives are saved in poor countries by focusing on the 'low hanging fruit' - those most readily reached by extending proven interventions through traditional service delivery modes. However, a review of evidence and experience conducted by UNICEF in mid-2011 $1^{2}$ demonstrates that this is not true. Excluded populations within countries often have higher fertility rates, and then experience higher rates of child mortality as they have less access to health interventions and greater exposure to risk than more affluent groups. Consequently, these populations have the greatest scope for gains in survival and development outcomes.

Equity involves recognising that people are different and require specific support and measures to overcome the

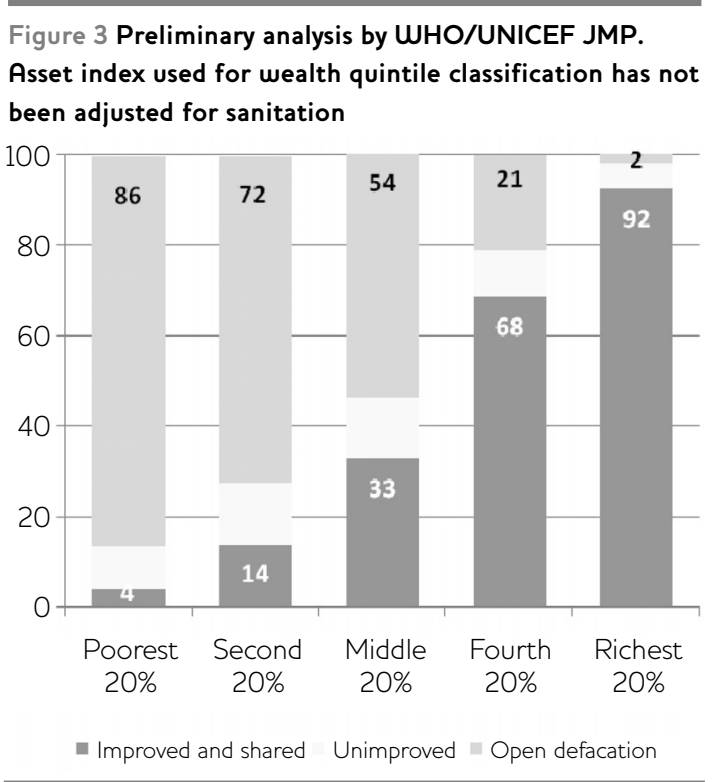

Source Population weighted average based on Bangladesh Demographic and Health Surveys (DHS) 2007, India National Family Health Survey (NFHS) 2006, Nepal DHS 2006, Pakistan DHS 2007, covering 93 per cent of the population in South Asia.

impediments that stand in the way of their being able to access and use services sustainably, in this case safe sanitation and adopting hygiene practices. It is about ensuring that services are accessible and affordable to all without discrimination. At a local level this would mean examining the context in which people live, work and play, and identifying the immediate barriers which stand in the way of people using hygienic toilets and washing their hands.

At higher administrative levels such as provincial, state or national levels, equity would be served by directing more resources to areas and communities with low sanitation coverage (geographic targeting) and to approaches which ensure that every individual has the means and responsibility to use and maintain sanitation facilities and wash their hands with soap, to promote their own and their neighbours' health and wellbeing (population targeting).

Equity principles must also apply in special situations that warrant special attention. Emergencies affect millions of people in South Asia every year: floods, droughts, earthquakes, landslides and civil strife displace large numbers of people for shorter or longer periods. Often, 


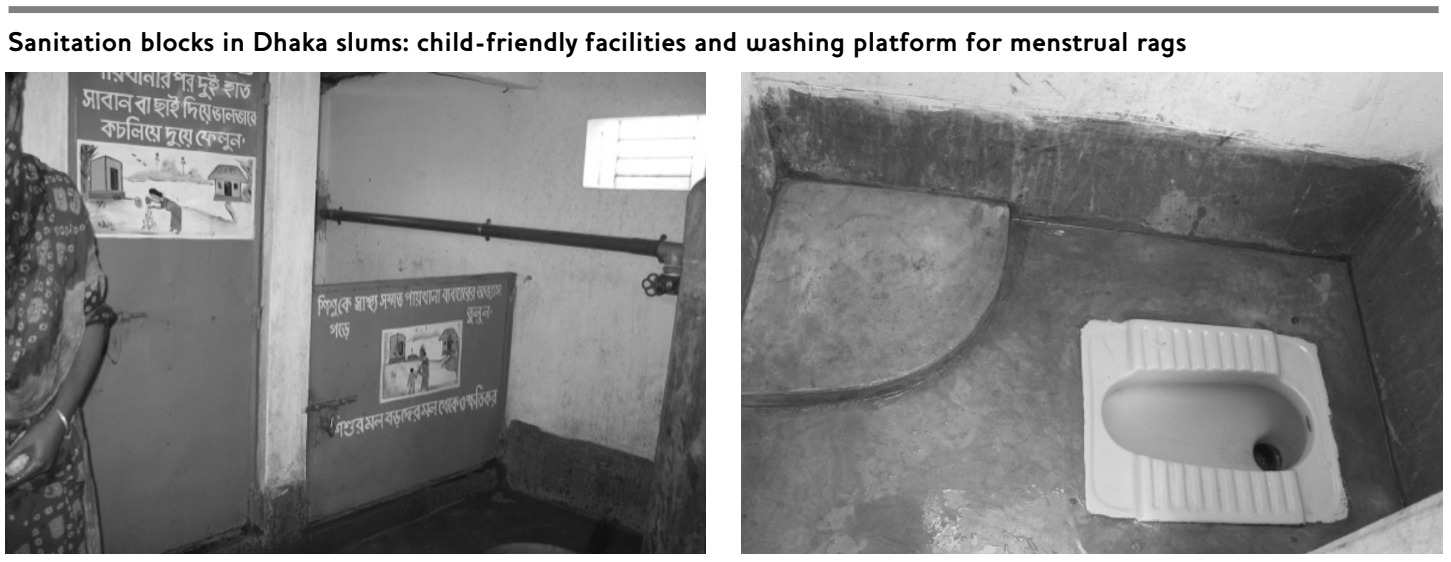

Photo credit: Archana Patkar, DFID Evaluation Mission of ASEH, Dhaka, 2006.

more than half of those displaced are children under the age of $18 .{ }^{3}$ With 58 per cent of the rural population practising open defecation, maintaining camps for the displaced free of open defecation and with adequate safe drinking water and water for hygiene practices is a major challenge. However, it is a fundamental right that cannot be denied.

On 30 September 2010, the UN Human Rights Council affirmed that the human right to water and sanitation is legally binding. ${ }^{4}$ This adds weight to the imperative to work towards full-scale coverage being sure to include everyone, even the poorest and most marginalised people. An initial impact assessment commissioned by the Department for International Development (DFID 2011) suggests that where countries have recognised the right to sanitation, trends towards accelerated progress can be noted. The findings are only conclusive for Bangladesh but suggest that it is highly probable that sanitation services, in countries which work towards fulfilment of the right to sanitation will be more equitable and inclusive than in those countries which have no specific rights focus.

\section{The way forward}

Action needs to be based on the principle of equity, which requires clear identification of and effective response around specific barriers in the following three categories, based on the social model of disability. This can be used to help identify poverty and disadvantage, analysing the relationship between social barriers and individual limitations (Burchardt 2004).

- Attitudinal barriers arise essentially from a lack of respect, which results in isolation, prejudice, stigma, misinformation and lack of self-confidence of those who are marginalised. They are also responsible for the disproportionate burden placed on women and girls in the region as de facto household managers of water, sanitation and hygiene.

- Environmental barriers impede physical accessibility to infrastructure and to communication; for example, toilet and squat pan designs which are difficult to use for the disabled, older people and pregnant women; and pans and traps that are improperly sized and daunting for young children.

- Institutional barriers cover a host of issues. Acts of omission, such as lack of specific policies for the excluded including finance, knowledge, skills and consultation mechanisms, and acts of commission such as administrative and financial corruption. Poor accountability mechanisms perpetuate weak governance, leaving officialdom blind to the deplorable conditions of the most marginalised groups.

Formidable though these barriers may seem, there are examples throughout the region of individuals and organisations that have overcome these barriers and brought about significant change through their passion, commitment, innovation and systems. There is therefore no reason why committed action, based on the principles of equity and inclusion, cannot work and on a significant scale.

The examples that follow are from different countries with their own governance systems and unique challenges. While each case study is unique it is possible to draw out common lessons from all of them that we will then seek to apply at scale. 


\section{Box 1 Including people with disabilities in Nepal}

About 2.9 million people in Nepal - approximately 10 per cent of the population - live with some form of impairment. The protection and promotion of their rights is enshrined in Article 13 of the current Interim Constitution of Nepal, and Article 26 proposes special provisions in health, education and social security. But the policies are poorly implemented and traditional attempts to increase coverage of sanitation have marginalised or excluded the needs of disabled people. After studying the barriers to latrine use faced by disabled people, WaterAid Nepal partner Nepal Water for Health (NEWAH) embarked on the 'Sanitation Access for Disabled People Project' with eight Village Development Committees in Baglung district. It supported families to address environmental barriers by adapting latrine designs to make them more accessible in a way that is suited to the terrain and local culture; helped to create a District Disabled Support Committee under the leadership of the District Development Committee to provide institutional support for programmes targeting disabled people; and employed advocacy activities to help sensitise the district and village development committees and other stakeholders to the needs of disabled people in development interventions. The workshops and media coverage of disability issues have also increased awareness among the public, influencing national policy and programmes. While this has shown what is possible, more work is needed to increase awareness, to monitor the disability provision in basic services, and to adapt sanitation designs. ${ }^{6}$

- Addressing discrimination and exclusion in schools: A UNICEF study looked at equity in school water and sanitation in Bhutan, Nepal, Bangladesh and India (UNICEF 2009). Inequity and discrimination are features of all societies and many of these are systematically reproduced in the school environment. The study found that adolescent girls faced disadvantage and stigma when they were menstruating, with many girls staying away from school for several days each month. Some schools also discriminated against different groups by selecting certain children to clean toilets, while others were made to sit separately at the back of the class. Ignorance and insensitivity towards children with special needs was widespread. On the other hand, the study also found examples of good practices in all four countries, which successfully address attitudinal, environmental and institutional barriers to inclusion. These are schools with good facilities, a shared understanding of responsibilities, equitable distribution of cleaning duties where no child feels exploited or singled out, and the active support of teachers who encourage an ethos of equality so that all children are accorded equal respect and dignity.

- Serving whole disadvantaged areas and special needs: In Bangladesh an innovative sanitation programme, Advancing Sustainable Environmental Health (ASEH), was designed with the explicit objective of working in the most disadvantaged parts of the country, where mainstream players such as the government would find it hard to work. Financed by DFID, WaterAid and its non-governmental organisation (NGO) partners chose the geopolitically disadvantaged hilly tribal areas characterised by stagnant swamps, annually flooded riverine deltas and dense urban slums with insecure tenure - to deliver services through a zero subsidy, Community-led Total Sanitation approach (see Kar, this IDS Bulletin). Building capacity, working with local governments, empowering people and learning along the way, ASEH has improved sanitation and hygiene practices for millions of poor people, influencing policy and practice for the sector overall. In Bangladesh, the local government (Union Parishad) earmarked funds to be used to promote sanitation, through both software activities and hardware subsidies targeted at the ultra poor. Under the programme, community ward members were free to allocate Union Parishad assistance for toilets as they saw fit. Not all of this assistance went to the ultra poor, and not all ultra poor households received help. The range of assistance offered by ASEH includes providing extremely low-cost and shared latrines, access to microfinance, and innovative, contextspecific approaches tailored to cultural and geographical needs (low-lying, hilly, coastal, geopolitically marginalised, etc.). All of these have been responsible for changing the 
Figure 4 Progress on increasing access to sanitation in India by health quintile since SACOSAN IV

\section{\% of households with access to toilet for each Wealth quintile as per special tabulation by UNICEF based on JMP-2010}

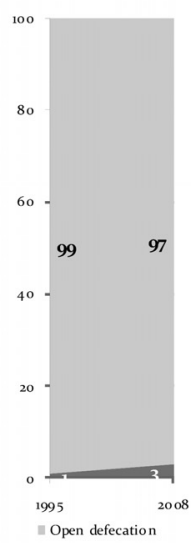

- Access to Sanitation

Poorest

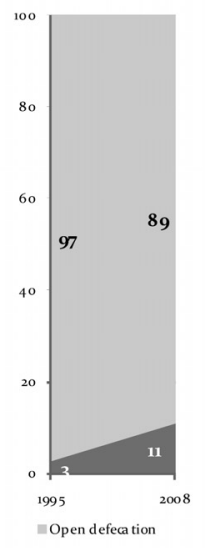

-Access to Sanitation

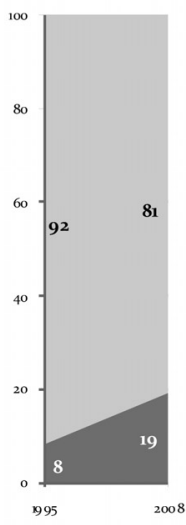

- Open de fecation

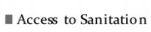

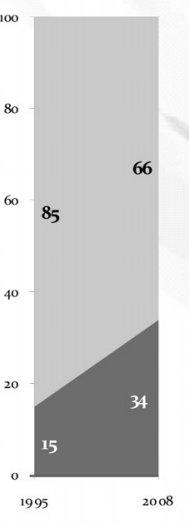

= Open defecation

-Access to Sa nitation

$4^{\text {th }}$

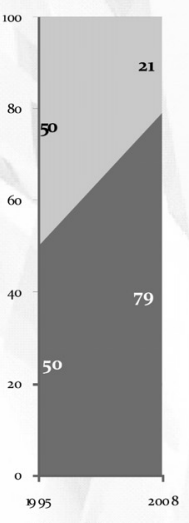

In Open defecation

- Access to Sanitation

Richest
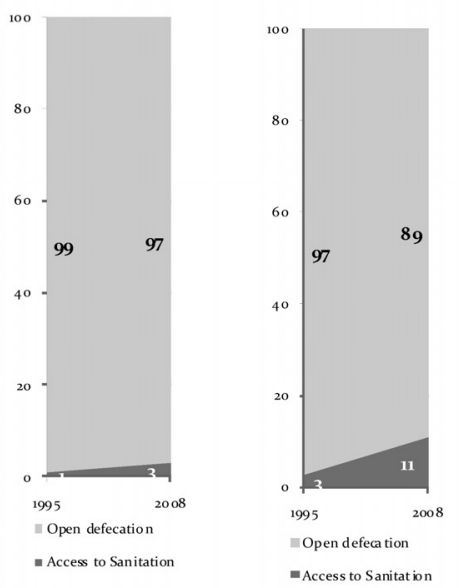

Poorest

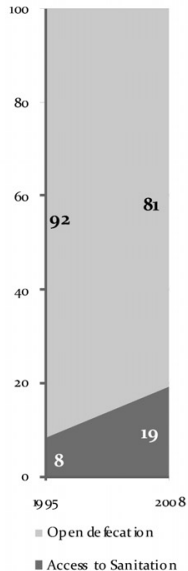

$3^{\text {rd }}$
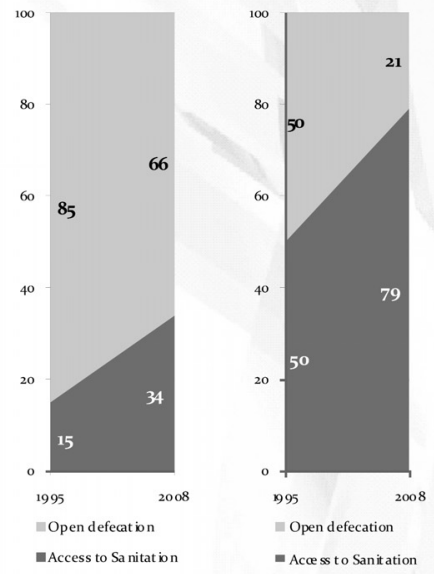

$4^{\text {th }}$ 
situation at scale, resulting in a reported 5.6 million sanitation beneficiaries and 6.8 million hygiene beneficiaries at the end of the five-year programme. ${ }^{5}$

The example in Box 1 shows how it has been possible to increase sanitation access to people with disabilities in Nepal. This approach can easily be incorporated into national sanitation strategies.

\section{Using the experience to mainstream equity and inclusion}

The following is a summary of the factors for success from a number of regional cases.

Experience shows that programmes that address institutional, environmental, and attitudinal barriers together can ensure access to sanitation and hygiene for even the most marginalised groups. To take the success to scale we have applied the principles that emerge from smaller scale programmes to the different elements of a national sanitation and hygiene strategy.

\subsection{Political commitment}

Countries in the region have made real strides regarding explicit policies that recognise exclusion and seek to address it in sanitation, as demonstrated by the examples below:

- Nepal (2007) - The interim Constitution states that all citizens are entitled to live in a clean and healthy environment;

- Pakistan (2006) - The needs of women, children and the disabled are given priority in all policy, planning and implementation processes;

- Bangladesh (1998) - Assigning priority to underserved and unserved areas, providing credit facilities for the poor to bear the costs of water and sanitation services, and measures will be taken so that users can bear increased cost of sanitation services. In the case of hard core poor communities, educational institutions, mosques and other places of worship, the costs may be subsidised partially or fully. In public toilets, separate provisions shall be made for women users. At the local level, 20 per cent of the Annual Development Plan is now earmarked for sanitation and there is evidence that this money is being used innovatively with far reaching impact.

- India Total Sanitation Campaign (modified guidelines) (2004) states that proper sanitation is one of the basic determinants of quality of life and the human development index. It is a human right to which every citizen is entitled. The concept of sanitation was, therefore, expanded to include personal hygiene, including menstrual hygiene and hand washing after defecation and before handling food, home sanitation, safe water, garbage disposal, excreta disposal and wastewater disposal.

- India Urban (2008) - says every urban dweller should be provided with minimum levels of sanitation, irrespective of the legal status of the land in which he/she is dwelling, possession of proof of identity or status of migration. At least 20 per cent of the funds under the sanitation sector should be earmarked for the urban poor. The issues of cross subsidies, the urban poor and their involvement in the collection of operation and maintenance charges should be considered.

\subsection{Monitoring}

Special attention to the identification of specific groups without access to sanitation and the attendant reasons would need to be accompanied by monitoring systems with disaggregated data to track changes at local, sub-national and national levels. If we can look at success from the lens of the vulnerable, we will make real progress. The analysis of Statistics and Monitoring Multiple Indicator Cluster Survey (MICS) and Demographic and Health Surveys (DHS) data by wealth quintile by the WHO/UNICEF Joint Monitoring Programme for Water and Sanitation $(\mathrm{JMP})^{8}$ is a good beginning, but we need to find better indicators to ensure monitoring focuses efforts and resources on those who are most difficult to reach at national and sub-national levels.

Figure 4 shows graphs presented by India's Ministry of Drinking Water \& Sanitation at a meeting of the Inter-country Working Group in Colombo, on 4 November 2010, to show progress since SACOSAN III. ${ }^{9}$

Efforts are already under way to translate the five pillars of the human right to sanitation - i.e. accessibility, affordability, adequacy, acceptability and safety - into global indicators that post-2015 recognise the excluded and most marginalised people in the measurement of achievement and success. More work is needed to institutionalise these across the sector. 


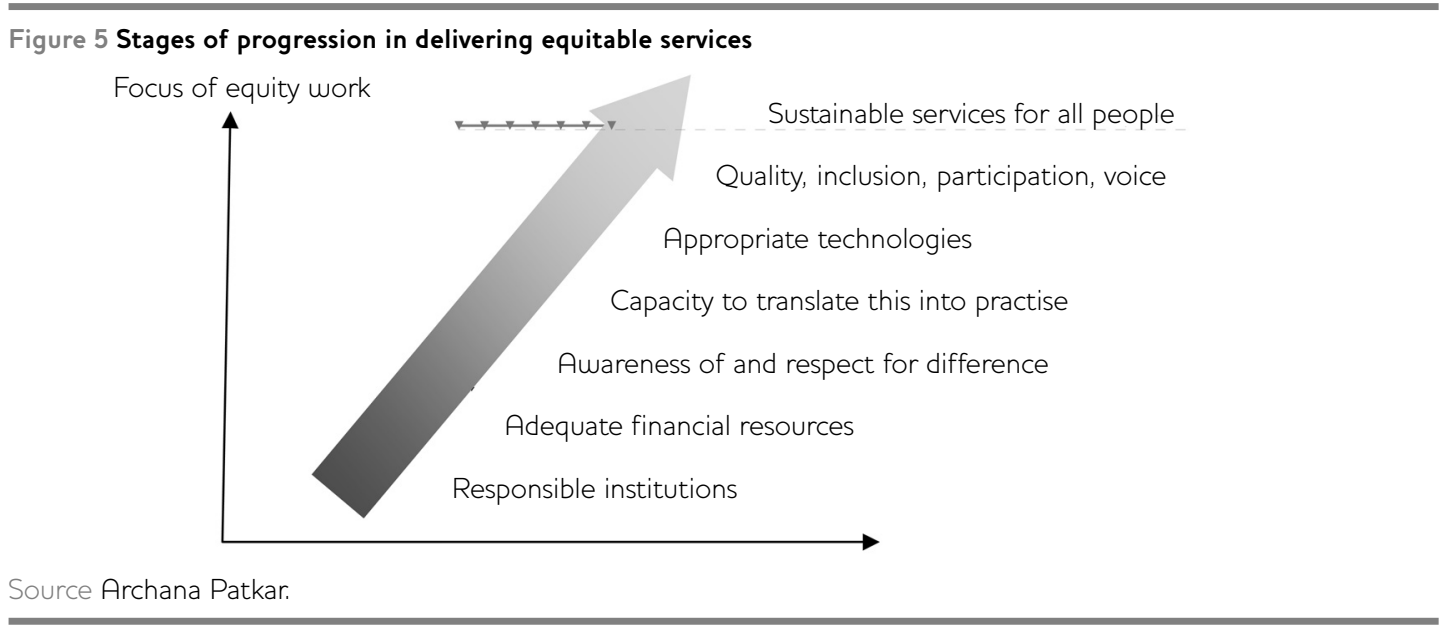

\subsection{Institutional structure and capacity}

A dedicated administrative structure/unit within governments for rural sanitation at all three levels (local, sub-national and national), with the mandate and means (both financial and human) to ensure equity and including fair representation by excluded groups, would enhance governments' capacity to understand and respond appropriately to issues of equity and inclusion. This has been demonstrated at scale by local governments in Bangladesh, and some states in India, among others in the region. ${ }^{10}$

\subsection{Approach to creating demand and scaling-up}

South Asia has the best and most widespread examples of total sanitation at scale, along with a range of projects targeting excluded groups. This learning has yet to be cross-fertilised to ensure that the sector works to minimum standards that ensure inclusive access and use for all. Wide dissemination of information about the right to sanitation, ensuring opportunities for the voices of the excluded in the exercise of demand creation, backed by earmarked finances to facilitate their participation, would be another step in their inclusion.

\subsection{Technology promotion and supply chain}

The promotion of appropriate technology options to meet the different needs of the excluded would be a significant step towards making inclusion a reality. Public sanitation should always include provision for menstrual hygiene management, disabled access, child friendly taps, pans and urinals, well located and functioning hand washing stations, adequate light and ventilation, safety and security.

\subsection{Finance and incentives}

All of the above steps depend on dedicated financial allocations for rural and urban sanitation in general (most countries in the region still lack a dedicated budget for sanitation) and earmarked allocation of funds for enabling all the above steps in particular. This would need to be accompanied by a system of incentives and sanctions for performance, approaches and results. The financial award system for 'open defecation-free' (ODF) status in Maharashtra state, which was later adopted by the Government of India as the Nirmal Gram Puruskar award, is one example.

The history, traditions, pace, structure and trajectory of political, social and economic development varies widely across the countries in South Asia. Any set of suggestions will need to be flexible enough to adapt to local situations and circumstances. In broad terms, however, one could track the progression to total and complete inclusive sanitation, access and usage, for all groups along an equity enabled graph as shown in Figure 5.

\subsection{Looking at services with an equity lens}

Figure 5 shows progression in delivering equitable services - starting with policies and commitments, matching these with institutions and investments, strengthening capacity to interpret policies with sensitive approaches and design, appropriate and cost-effective hardware to match user needs, information and widespread awareness around rights and how these can be accessed, and strong demand at every level, every time to eradicate exclusion. 


\section{Box 2 Including people living with HIV/AIDS in Uttar Pradesh, India}

People living with HIV/AIDS (PLHA) are the most discriminated against in society, economically, socially and psychologically. The immuno-compromised status of PLHA renders them more susceptible to opportunistic WASH-related infections like diarrhoea, which is experienced by over 90 per cent of patients with AIDS. It becomes more frequent as immune deficiency progresses. Easily accessible and sufficient water and sanitation are indispensable for people living with HIV/AIDS as well as for the provision of home-based care to AIDS-affected persons. In 2008, WaterAid India entered into partnership with Uttar Pradesh State AIDS Control Society for a project, entitled Programme on Arresting Opportunistic Infections for People Living with HIV/AIDS, to help improve the quality of their lives through water and sanitation. The project is being implemented through CREATE in 14 districts and involves antiretroviral therapy cells, which are care and support centres. These have also become WASH information centres where people living with HIV/AIDS are able to learn about key hygiene practices. The centres share information through posters and pamphlets, display different toilet models, and offer a range of audio, video and other materials. As well as group and individual counselling, PLHAs are also able to use good quality facilities at the centres, such as water filters, washbasins, urinals and latrines. Staff members are trained on WASH issues and are able to tell PLHAs about the importance of good WASH practices in their lives. ${ }^{7}$

\section{Recommendations}

There is widespread recognition that the gains of the past decade have not accrued to those who need them most and that future interventions cannot continue with business as usual. This article proposes the application of an equity and inclusion lens to existing monitoring frameworks covering the range of stakeholder, policy and practice dimensions. Suggested criteria to assess commitment, capacity and practical options for ensuring and benchmarking equity and inclusion in sanitation are presented below.

\section{Political commitment and strategy for implementation}

a Constitutional guarantees for equality, recognising complex nature of exclusion in sanitation and hygiene;

b Right to sanitation and hygiene enabled by the availability of adequate water at household level, should be explicit in policy;

c Sanitation and hygiene strategy includes detailed analysis of context-specific inequalities and exclusions in relation to sanitation and hygiene;

d This political commitment to sanitation and hygiene explicit in party manifestos, annual sector reviews and mid-term appraisals.

\section{Monitoring}

a Focus on those who do not practise sanitation and hygiene and why - at national, subnational and local level; b Evaluations of sanitation and hygiene programmes, use disaggregated data and report on equity and inclusion in findings;

c Monitoring methods include independent monitoring of use and practice, equity focused national monitoring systems and sample surveys to gauge progress, public hearings, citizen report cards and reviews, and independent reviews of services for particular groups.

\section{Institutional structure and capacity}

a Dedicated mandate and unit for sanitation, with specific responsibility and performance standards for equity and inclusion;

b Representation of excluded groups at all levels of institutions - gender, disability, religion, caste;

c Capacity to understand and respond to equity and inclusion.

\section{Approach to creating demand and scaling-up}

a Ensuring that the voice of excluded groups is amplified in the demand for sanitation and hygiene and in designing inclusive facilities;

b Financing mechanisms to enable the participation of disadvantaged groups;

c Information about the right to sanitation is known and understood by disadvantaged groups.

\section{Technology promotion and supply chain}

a Technology options include diverse solutions for different needs (gender, disability, age, seasons, soil); 
b Options are affordable for the poorest;

c Public sanitation facilities always include water for hand washing, facilities for menstrual hygiene management and disabled access.

\section{Finance and Incentives}

a Budget allocation or earmarking for participation of excluded groups and inclusive design;

b Sanctions for non-inclusion;

c Money flows to whom it is intended and is tracked and regularly shared with the public.

\section{Conclusion}

The journey to ensure accountability and results for the poorest is a long one. In September 2010, by a vote of 122 in favour to none against, with 41 abstentions, the General Assembly of the United Nations adopted a resolution calling on states and international organisations to provide financial resources, build capacity and transfer technology, particularly to developing countries, in scaling-up efforts to provide safe, clean, accessible and affordable drinking water and sanitation for all. The General Assembly expressed deep concern that some 884 million people were without access to safe drinking water and more than 2.6 billion lacked access to basic sanitation. Bearing in mind the commitment to fully achieve the MDGs, it expressed alarm that 1.5 million children under five years old died each year as a result of waterand sanitation-related diseases, acknowledging that safe, clean drinking water and sanitation were integral to the realisation of all human rights.

In South Asia this has been acknowledged. Following SACOSAN IV (for which this article was developed), the Colombo Declaration of 7 April

\section{Notes}

1 See www.un.org/ga/search/view_doc.asp? symbol=A/RES/64/292

2 A special report on a study by UNICEF (2010), Narrowing the Gaps to Meet the Goals, shows that an equity-focused approach to child survival and development is the most practical and cost-effective way of meeting the health MDGs for children, www.unicef.pt/ docs/Narrowing_the_Gaps_to_Meet_the_Goal s_090310_2a.pdf (accessed 13 January 2012).

3 See www.unicef.org/protection/index_armed conflict.html; www.savethechildren.org.uk/ en/32_1300.htm; www.unicef.org/emerg/
2011 acknowledged the gravity of the sanitation situation in South Asia and renewed its commitment to delivering sustainable sanitation and hygiene to all (SACOSAN 2011). It recognised the need to reflect the UN resolution on the right to sanitation in legislation and to realise this in programmes. It spelt out the need to design and deliver context-specific equitable and inclusive sanitation and hygiene programmes including better identification of the poorest and most marginalised groups in rural and urban areas. This includes transparent targeting of financing to programmes for those who need them most, and raising the profile of WASH in schools with the objective of ensuring that every new and existing school at every educational level has functioning, child-friendly toilets, separate for girls and boys, with facilities for menstrual hygiene management. A key regional commitment that is now being taken forward by the Inter-country Working Group is to develop harmonised monitoring mechanisms with roles and responsibilities clearly defined, using agreed common indicators which measure and report on processes and outcomes at every level, including households and communities. These should allow for disaggregated reporting of outcomes for marginalised and vulnerable groups.

This commitment is welcome but more is needed. Without explicit attention to equity, progress will continue to be discriminatory and inequitable. Articulation of the right to sanitation must be backed by corresponding measures to ensure gains for the poorest and most vulnerable. The examples given in this article show that this can be achieved in different contexts. Equity is not just a good idea but is, in fact, a basic tenet and mandate in policy and practice to achieve sanitation for all.

haiti_52590.html (accessed 1 January 2011).

4 See http://daccess-dds-ny.un.org/doc/UNDOC/ GEN/G10/166/33/PDF/G1016633.pdf?Open Element

5 ASEH (April 2009) End of Project Evaluation Study, Final Report (Rural and Urban Component) Participatory Management Initiative for Development (PMID) and Bangladesh Centre for Advanced Studies. 6 'Creating User-Friendly Water and Sanitation Services for the Disabled: The Experience of WaterAid Nepal and its Partners', WaterAid 2008.

7 See www.irc.nl/page/53176 
8 'A Snapshot of Sanitation in South Asia with a Focus on Inequities', WHO/UNICEF Joint Monitoring Programme for Water Supply and Sanitation (JMP) - prepared for SACOSAN IV (forthcoming), www.unicef.org/statistics/ index_24302.html.

9 The graphs use an income and expenditure measurement to classify households by wealth quintiles. This classification is not exactly comparable to the asset-based index of the NFHS surveys.

10 The Bangalore Water Supply and Sewerage Board has a dedicated Social Development Unit with the mandate of reaching the slums.

\section{References}

Burchardt, Tania (2004) 'Capabilities and Disability: The Capabilities Framework and the Social Model of Disability', Disability \& Society 19.7: 735-51(17), December

DFID/Coffey International Development (2011) Assessing the Impact of a Right to Sanitation on Improving Levels of Access and Quality of Services, Executive Summary, April, London: Department for International Development

SAGOSAN (2011) The Colombo Declaration, Fourth South Asian Conference on Sanitation, 4-7 April 2011, www.sanitationdrive2015.org/
The tripartite partnership between the Dhaka Municipal Water and Sanitation Authority (DWASA), the Municipal Corporation responsible for human settlement and land tenure, and the local NGO DSK is a model replicated many times over to deliver services to the urban poor without tenure in a costeffective and equitable manner. The role of supporting ministries and departments of health, education and social welfare is also critical in delivering quality services, particularly in schools and health clinics/hospitals.

endorsements/ fourth-SouthAsian-conferenceon-sanitation_04-2011.pdf (accessed 20 December 2011)

UNICEF (2009) Equity in School Water and Sanitation: Overcoming Exclusion and Discrimination in South Asia, UNICEF Regional Office for South Asia

WSP (2011) The Economic Impact of Inadequate Sanitation in India, New Delhi: Water and Sanitation Program, www.wsp.org/wsp/sites/ wsp.org/files/publications/WSP-esi-india.pdf (accessed 13 January 2012) 